\title{
Model for Optimal Management of the Spare Parts Stock at an Irregular Distribution of Spare Parts
}

\author{
Svetozar Madzhov \\ Forest Research Institute, Bulgarian Academy of Sciences, Sofia 1756, Bulgaria
}

\begin{abstract}
The article presents a model for optimal management of the SP (Spare Parts) stock when they are irregularly distributed. The SPs are distributed irregularly and the supply and purchase costs depend on the number of elements. It is necessary to fix the optimal number of supplies, for which the total purchase and storage are minimal.
\end{abstract}

Key words: SP, optimum management, stock, forestry.

\section{Introduction}

The question of the optimal stock management has been discussed in one way or another in many publications [1-7]. Most often, mono nomenclature determinant models are discussed. They are of interest, because they enable us to study the ideology of research methods used in more sophisticated systems. Practically, a large number of different types of stocks are stored in the warehouses, which imposes that different kinds of nomenclature models for stock management of SP (Spare Parts) are developed. Another important feature of the SP management system in the practice is the presence of restrictions, more total number of deliveries during the year, the maximal storage space, maximal volume of each separate delivery, minimal quantity of the ordered production, etc.

The companies in the real economy usually do not dispose of unlimited financial resource, to be invested in stock, but just the opposite-they dispose of a limited working capital, by which they maintain the stock. The current model reflects the irregularity of forestry production, i.e. the fact that the mechanization load, respectively the need of SP is high, and the

Corresponding author: Svetozar Madzhov, Assistant Professor, Ph.D., research field: Forest research. purchase costs and stock fill costs depend on the number of elements. This is a common situation, especially in today's market conditions, when supply depends largely on market demand.

It is necessary to fix the optimal number of supplies, for which the total costs for stock purchase and storage are minimal.

\section{Materials and Methods}

The current model reflects the irregularity of forestry production, i.e. the fact that the mechanization load, respectively the need of SP is high, and the purchase costs and stock fill costs depend on the number of elements. This is a common situation, especially in today's market conditions, when supply depends largely on market demand.

It is necessary to fix the optimal number of supplies, for which the total costs for stock purchase and storage are minimal.

If the storage function of SP is $Q \mathrm{~m}(t)$, then the cost of $Q$ elements for the period $\left[t_{1}, t_{2}\right]$ can be calculated by the Eq. (1):

$$
Q=\int_{t 1}^{t 2} Q m(t) d t
$$

If in the timeframe $t_{0}=0$ till $t_{\mathrm{n}}$ the stock is filled $n$ times through equal time intervals $\Delta t_{i}=t_{n} / n$ and in the moment: 


$$
\begin{aligned}
& t_{0}=0 \\
& t_{1}=t_{n} / n ; \\
& t_{2}=2 t_{n} / n ; \\
& \ldots \ldots ; \\
& t_{n-1}=(n-1) t_{n} / n ; \\
& t_{n} .
\end{aligned}
$$

If at the moment $t_{0} Q_{1}$ elements are purchased, then at the moment $t_{\mathrm{i}}$ the number of the remaining unused elements according to Eq. (1) will be:

$$
Q_{1}-Q\left(t_{i}\right)=Q_{1}-\int_{t 1}^{t 2} Q m(t) d t
$$

Let us designate with $S$ the costs of storage of one element for the whole period from $t_{0}$ till $t_{\mathrm{n}}$ and we receive, that for the time $\Delta t_{i}=t_{n} / n$ the storage costs of one element will be $\frac{S}{n}=S . \Delta t_{i} / t_{n}$. Then according to Eq. (2), the storage costs of the whole volume of elements for the time from $t_{0}$ till $t_{\mathrm{n}}$ are:

$$
\frac{S}{t_{n}} \int_{t 0}^{t 1}\left[Q_{1}-Q(t)\right] d t=\frac{S}{t_{n}}\left[Q_{1}\left(t_{1}-t_{0}\right)-\int_{t 0}^{t 1} Q(t) d t\right]
$$

If at the moment $t_{\mathrm{i}}$ all elements have been used up, i.e. $Q\left(t_{1}\right)=Q_{1}$, then we purchase additional quantity of elements $/ \mathrm{Q}_{2}-\mathrm{Q}_{1} /$ and similarly the storage costs for the period $\left(t_{1}, t_{2}\right)$ will be:

$$
\frac{S}{t_{n}} \int_{t 1}^{t 2}\left[Q_{2}-Q(t)\right] d t=\frac{S}{t_{n}}\left[Q_{2}\left(t_{2}-t_{1}\right)-\int_{t 1}^{t 2} Q(t) d t\right]
$$

Till the moment $t_{2}$ all elements have been used up, i.e. $Q\left(t_{2}\right)=Q_{2}$ etc..

Therefore the storage costs $C_{1}$ for the whole period $\left(0, t_{\mathrm{n}}\right)$ will be:

$$
\begin{gathered}
C_{1}=\frac{S}{t_{n}}\left[\sum_{i=1}^{n} Q\left(t_{i}\right)\left(t_{i}-t_{i-1}\right)-\sum_{i=1}^{n} \int_{t_{i-1}}^{t_{i}} Q(t) d t\right]= \\
\frac{S}{t_{n}}\left[\sum_{i=1}^{n} Q\left(t_{i}\right) \Delta t_{i}-\int_{t_{0}}^{t_{i}} Q(t) d t\right]
\end{gathered}
$$

The purchase costs of i-s batch of SP with a volume $P_{\mathrm{I}}$ may be presented by $k+l P_{i}$, where $k$ are organizational costs (business trip costs, travel expenses, etc.), which are not dependant on the number of the elements. Therefore the costs $C_{2}$ for elements purchase are:

$$
\begin{aligned}
C_{2} & =k+l Q\left(t_{1}\right)+k+l\left[Q\left(t_{2}\right)-Q\left(t_{1}\right)\right]+\ldots \ldots . \\
& +k+l\left[Q\left(t_{n}\right)-Q\left(t_{n-11}\right)\right]=k n+l Q\left(t_{n}\right)
\end{aligned}
$$

Then the total costs $C$ are:

$$
\begin{gathered}
C=C_{1}+C_{2}=\frac{S}{t_{n}} \sum_{i=1}^{n} Q\left(t_{i}\right) \Delta t_{i}+k n+B= \\
\frac{S}{n} \sum_{i=1}^{n} Q\left(t_{i}\right)+k n+B
\end{gathered}
$$

where as,

$$
B=l Q\left(t_{n}\right)-\frac{\mathrm{S}}{\mathrm{t}_{\mathrm{n}}} \int_{t_{0}}^{t_{n}} Q(t) d t
$$

As $t_{0}=0$ and $t_{\mathrm{n}}=$ const, then $B$ is invariable, which is not dependant on $n$ and in order to find the smallest value of the total costs it is enough to study the equation:

$$
P(n)=\frac{S}{n} \sum_{i=1}^{n} Q\left(t_{i}\right)+k n
$$

at extreme.

In this case, we have to note that $Q\left(t_{i}\right)$ depends on $n$, because $t_{i}=t_{n} / n$. Therefore after we find out the meaning of $n$, we can determine the necessary volume of the purchased elements by the following formulas:

$$
\begin{aligned}
& Q_{1}=Q\left[t_{n} / n\right] \\
& Q_{2}-Q_{1}=Q\left[2 t_{n} / n\right]-Q\left[t_{n} / n\right] \\
& \ldots \ldots \ldots . ; \\
& Q_{n}-Q_{n-1}=Q\left[t_{n} / n\right]-Q\left[(n-1) t_{n} / n\right] .
\end{aligned}
$$

We assume that the function $Q_{m}(t)$ looks like:

$$
Q_{m}(t)=a+b t+\gamma t^{2}
$$


As the researches done in Ref. [1] show that functions of this kind are applicable to machines with an operating life $t_{e} \geq 3$ years. The presented model and the relation Eq. (9) allow defining the optimal delivery number on the basis of variable real values of SP demand, presumed that the costs of purchase and storage of stock are minimal. According to Eq. (1), we receive for the cost of elements $Q(t)$ :

$$
Q(t)=\int_{0}^{t} Q m(t) d t=a t+\frac{b t^{2}}{2}+\frac{\gamma t^{3}}{3}
$$

In compliance to Eq. (7) $P_{n}$ is equal to:

$P(n)=\frac{S . t_{n}}{n^{2}}\left[a \sum_{i=1}^{n} i+\frac{b t_{n}}{2 n} \sum_{i=1}^{n} i^{2}+\frac{\gamma t_{n}^{2}}{3 n^{2}} \sum_{i=1}^{n} i^{3}\right]+k n$

It is known that

$$
\begin{gathered}
\sum_{i=1}^{n} i=\frac{n(n+1)}{2} ; \quad \sum_{i=1}^{n} i^{2}= \\
\frac{n(n+1)(2 n+1)}{6} ; \quad \sum_{i=1}^{n} i^{3}=\frac{n^{2}(n+1)^{2}}{4}
\end{gathered}
$$

Then

$$
\begin{gathered}
P_{n}=\left(\frac{S . t_{n}}{2}\right)\left(a+\frac{b t_{n}}{6}\right)+\left(\frac{S . t_{n}}{2 n}\right)\left(a+\frac{b t_{n}}{4}+\frac{\gamma t_{n}^{2}}{6}\right) \\
+\left(\frac{S \cdot t_{n}^{2}}{12 n^{2}}\right)\left(b+\gamma t_{n}\right)+k n
\end{gathered}
$$

as $t_{n}=$ const, then the first addend does not depend on $n$ and it is enough to minimize the equation,

$$
R(n)=k n+\frac{l}{n}+\frac{d}{n^{2}}
$$

where

$$
l=\left(\frac{S \cdot t_{n}}{2}\right)\left(a+\frac{b t_{n}}{4}+\frac{\varkappa_{n}^{2}}{6}\right) \quad u \quad d=\left(\frac{S \cdot t_{n}^{2}}{12}\right)\left(b+\varkappa_{n}\right) .
$$

\section{Results and Discussion}

In study of Eq. (9), we found out that the optimal number of deliveries $(n)$ during the timeframe $\left(t_{n}\right)$ for the presented levels of storage costs $(S)$. The results of this study are illustrated in Fig. 1.

In study of Eq. (9), we found out that the optimal number of deliveries $(n)$ in the logistic system depends on the initial costs $(k)$. The optimal values are presented in Fig. 2.

We differentiate Eq. (9) and get:

$$
\begin{aligned}
& \frac{d R}{\partial n}=k-\frac{l}{n^{2}}-\frac{2 d}{n^{3}} ; \\
& \frac{d^{2} R}{\partial n^{2}}=\frac{2 l}{n^{3}}+\frac{6 d}{n^{4}} .
\end{aligned}
$$

We equate to zero and get a cubic equation $k n^{3}-\ln -2 d=0$ (10), which can be easily solved analytically.

The positive roots of Eq. (10) correspond to the extreme of Eq. (7). If $n$ is not an integer, we round it up to a whole. If the function has an extreme, it is minimal. We need to check whether $\frac{d^{2} R}{\partial n^{2}}>0$ and if so, then the function has a solution. To be able to solve the cubic Eq. (10) we set $-l / k=p ;-2 d / k=q$ and then the equation obtains the outlook:

$$
n^{3}+p n+q=0
$$

Eq. (11) is solved by the Cardan equations for a cubic equation. In our case, it is most convenient to work with the trigonometric solution.

Three cases can be considered depending on $Q=\left(\frac{p}{3}\right)^{3}+\left(\frac{q}{2}\right)^{2}$ :

(1) At $Q<0$ and $p<0$ Eq. (11) is solved under the formulas:

$$
y_{1}=2 \sqrt{-\frac{p}{3}} \cos \frac{\alpha}{3}, \quad y_{2}=2 \sqrt{-\frac{p}{3}} \cos \left(\frac{\alpha}{3} \pm \frac{\pi}{3}\right),
$$

$$
\cos \alpha=-\frac{q}{2 \sqrt{-\left(\frac{p}{3}\right)^{3}}}
$$




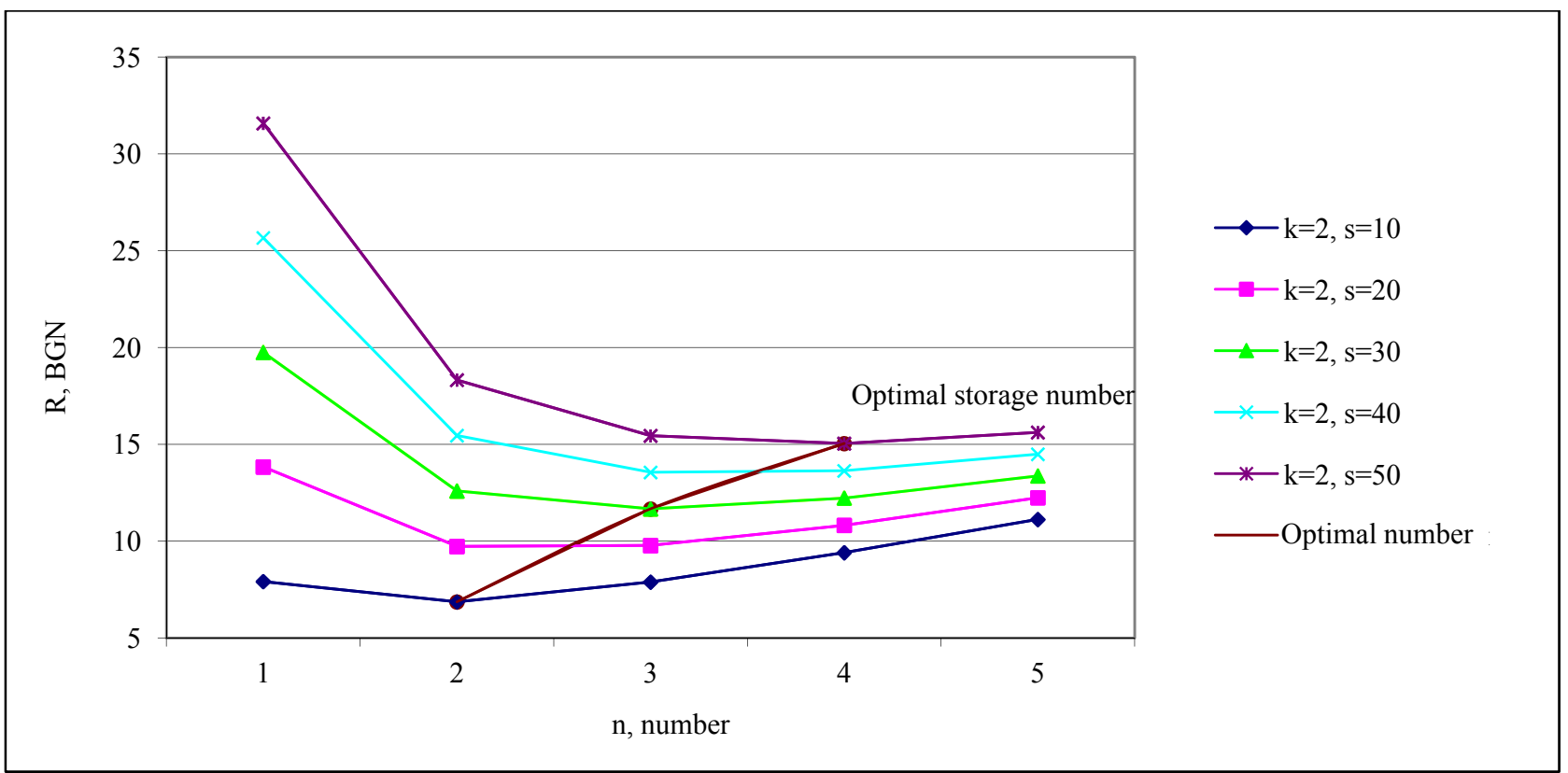

Fig. 1 Optimal number of deliveries $(n)$ in the logistic system depending on the storage costs $(S)$.

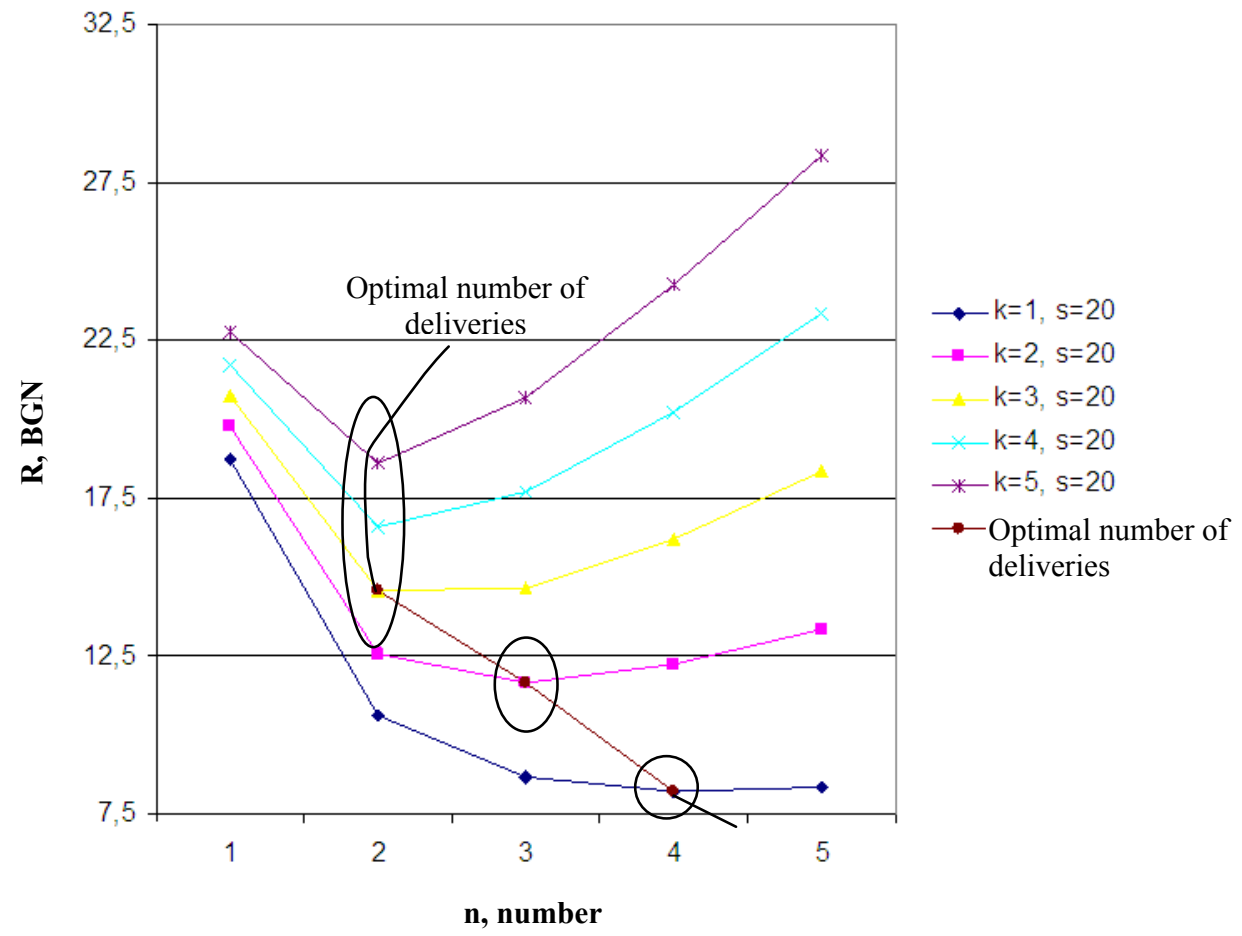

Fig. 2 Optimal number of deliveries (n) in the logistic system depending on the initial costs (к). 
(2) At $Q \geq 0$ and $p>0$ Eq. (11) is solved like this:

$y_{1}=-2 \sqrt{\frac{p}{3}} \operatorname{ctg} 2 \alpha, \quad y_{2,3}=\sqrt{\frac{p}{3}}(\operatorname{ctg} 2 \alpha \pm i \sqrt{3} \operatorname{cosec} 2 \alpha)$;

$\operatorname{tg} \alpha=\sqrt[3]{\operatorname{tg} \frac{\beta}{2}}\left(|\alpha| \leq \frac{\pi}{4}\right), \operatorname{tg} \beta=\frac{2}{q} \sqrt{\left(\frac{p}{3}\right)^{3}}\left(|\beta| \leq \frac{\pi}{2}\right)$

(3) At $Q \geq 0$ and $p<0$ Eq. (11) is solved like this:

$$
\begin{aligned}
& y_{1}=-2 \sqrt{-\frac{p}{3}} \operatorname{cosec} 2 \alpha, \\
& y_{2,3}=\sqrt{-\frac{p}{3}}(\operatorname{cosec} 2 \alpha \pm i \sqrt{3} \operatorname{ctg} 2 \alpha) ; \\
& \operatorname{tg} \alpha=\sqrt[3]{\operatorname{tg} \frac{\beta}{2}}\left(|\alpha| \leq \frac{\pi}{4}\right), \\
& \sin \beta=\frac{2}{q} \sqrt{\left(-\frac{p}{3}\right)^{3}}\left(|\beta| \leq \frac{\pi}{2}\right) .
\end{aligned}
$$

Eq. (11) is solved and its positive root is the requested value of $n$.

Let us now study the case, in which the function $Q_{m}(t)$ looks like:

$$
Q_{m}(t)=A e^{a t_{i}} .
$$

According to Eq. (1) for elements costs $Q(t)$ we get:

$$
Q(t)=\int_{0}^{t} Q m(t) d t=\frac{A e^{a t_{i}}}{a}
$$

In compliance with Eq. (6) $P_{n}$ looks like:

$$
P(n)=\frac{S}{n} \sum_{i=1}^{n}\left(\frac{A}{a} e^{a t_{i}}\right)+k n
$$

Considering that $e^{a t_{i}}=\cos a+t_{i} \sin a$, then:

$$
P(n)=\frac{S A}{n a} \sum_{i=1}^{n}\left(\cos a+t_{i} \sin a\right)+k n
$$

Given the fact that $\Delta t_{i}=t_{n} / n$, then:

$$
P(n)=\frac{S \cdot A}{n a}\left[\cos a+\frac{t_{n} \sin a}{n} \sum_{i=1}^{n} i\right]+k n
$$

It is known that

$$
\sum_{i=1}^{n} i=\frac{n(n+1)}{2}
$$

Then

$P(n)=\frac{S \cdot A}{n a}\left[\cos a+\frac{t_{n} \sin a}{2}\right]+\frac{s A t_{n} \sin a}{2 a}+k n$,

Given the fact that $t_{n}=$ const, then the second addend does not depend on $n$ and it is enough to minimize the equation:

$$
R(n)=\frac{q}{n}+k n
$$

where $q=\left(\frac{s \cdot A}{a}\right)\left(\cos a+\frac{t_{n} \sin a}{2}\right)$.

We differentiate Eq. (13) and we get:

$$
\frac{d R}{\partial n}=k-\frac{q}{n^{2}}
$$

$$
\frac{d^{2} R}{\partial n^{2}}=\frac{2 q}{n^{3}} .
$$

Equated to zero and we get a squared equation

$$
k n^{2}-q=0
$$

which is solved ana lytically.

The positive roots of Eq. (14) correspond to the extreme of Eq. (7). If it is assumed that $n$ is not a whole number, then it is rounded to the whole. If the function has an extreme, it is minimal. It is necessary to check whether $\frac{d^{2} R}{\partial n^{2}}>0$, and if so, then the function is resolved. The equation root is got after rework and $n=\sqrt{\frac{q}{k}}, \quad$ where $q=\left(\frac{s \cdot A}{a}\right)\left(\cos a+\frac{t_{n} \sin a}{2}\right)$.

After substituting $q$ in formula for $n$, we get: $n=\sqrt{\frac{2 s A \cos a+s A t_{n} \sin a}{2 a k}}$ 
In order that the equation has real positive roots, we have to prove that $\frac{d^{2} R}{\partial n^{2}}>0$, i.e. $\frac{d^{2} R}{\partial n^{2}}=\frac{2 q}{n^{3}}>0$. In order that the preceding equation is $>0$, it is necessary $q>0$ and $k>0$, but $k$ is positive by condition.

Further below we will study the fluctuation of the total costs $\left(R_{n}\right)$, depending on the model parameter, namely the delivery number $(n)$ during the period $\left(t_{n}\right)$. The object of the study is the fluctuation of the model parameter number of deliveries $(n)$ depending on the management factors and coefficient of the storage function $Q_{m}(t)=a+b t+t^{2}$. The management factors are the storage factors $(s)$, the initial costs $(k)$ and the storage function $\left(Q_{m}\right)$. The coefficients of the storage functions $\left(Q_{m}\right)$ are $a, b$ and $\gamma$.

After the total costs function rework we equate to zero the first derivative of the function and get a cubic equation, in which positive roots coincide to the extreme of the function. The three roots of the cubic equation are located at the cross point of the graphic with the ordinate $R_{n}=0$. The cubic equation has one positive and two negative roots, one of which has negative values close to zero. The positive root is the solution of the equation and gives the value of the deliveries' number $(n)$ during the period depending on the management factors, and the negative values of deliveries' number $(n)$ have no physical meaning.

Fig. 3 presents the fluctuation of supply number $(n)$ during the period depending on the coefficient values (a) by the stock function $\left(Q_{m}\right)$. The result analysis shows that with the increase of the coefficient $(a)$ the number of deliveries during the year $(n)$ grows quickly, i.e. the value of the coefficient $(a)$ influences strongly the deliveries number during the year $(n)$. The data for obtaining the coefficients of the regression equation ( $a$, $b, \gamma)$ are from direct observations.

The study, led with different values of the coefficient (b) (Fig. 4), over the deliveries number $(n)$, shows that the coefficient value $(b)$ of the stock function $\left(Q_{m}\right)$ influences insignificantly over the delivery number fluctuation during the year $(n)$.

The study, conducted by different values of delivery number $(n)$ at different values of the coefficient $(\gamma)$ of the stock function $\left(Q_{m}\right)$ (Fig. 5), proves that the coefficient value $(\gamma)$ practically does not influence the deliveries number $(n)$.

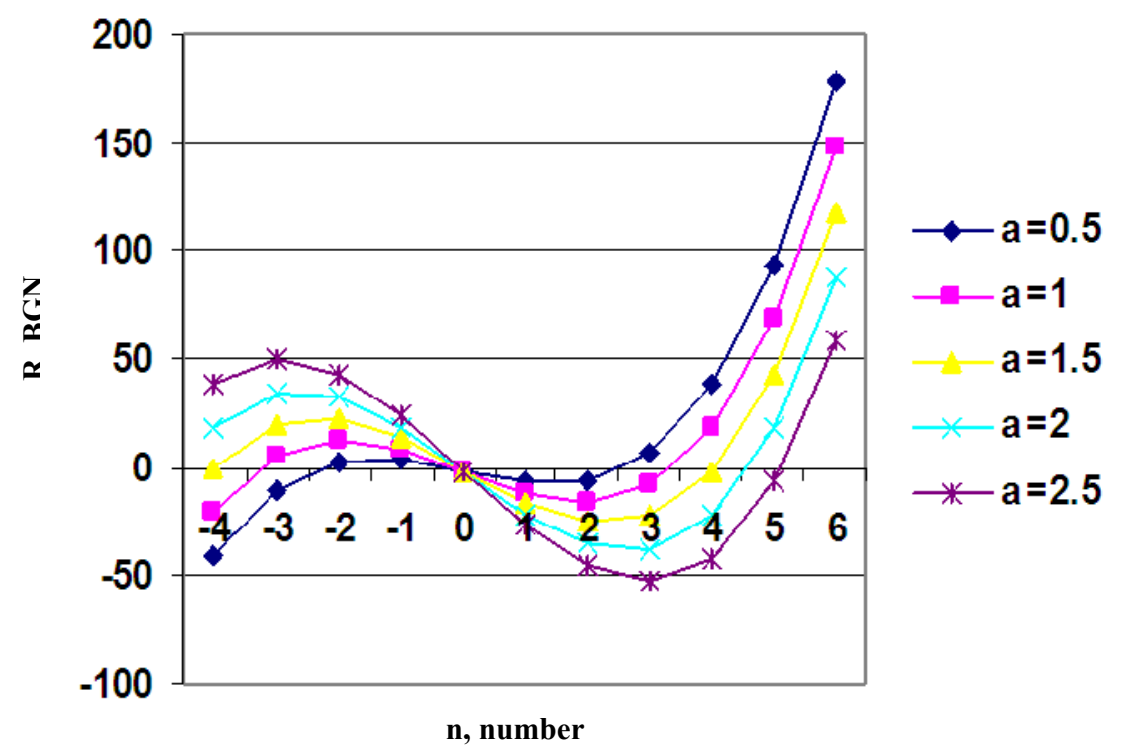

Fig. 3 The deliveries number (n) fluctuation during the period depending on the coefficient fluctuation (a) by the stock function $\left(Q_{m}\right)$. 


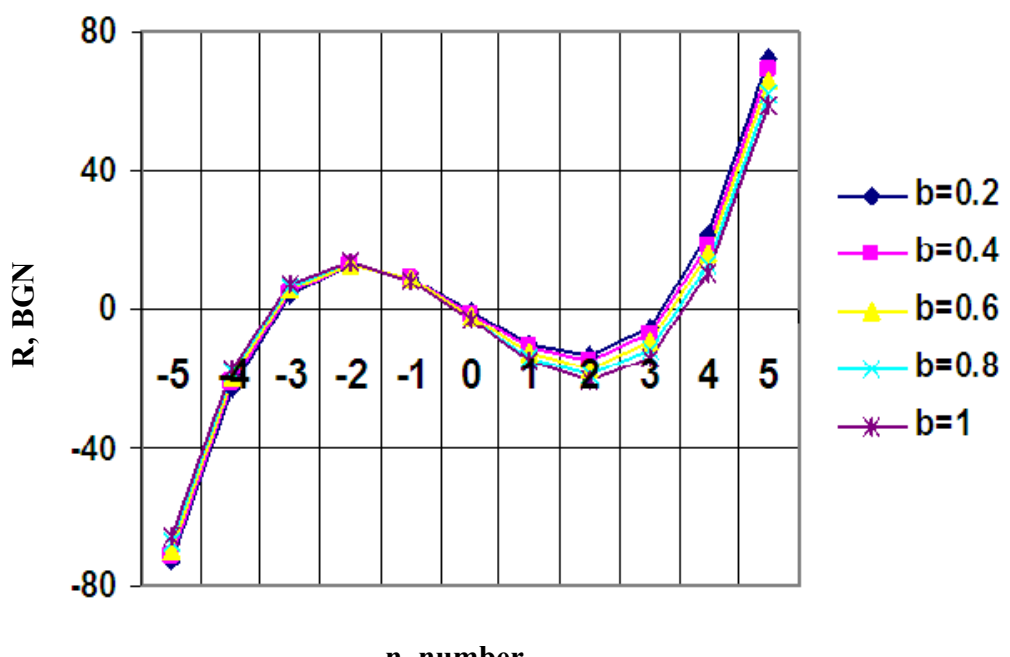

Fig. 4 The deliveries number $(n)$ fluctuation during the period depending on the coefficient fluctuation (b) by the stock function $\left(Q_{m}\right)$.

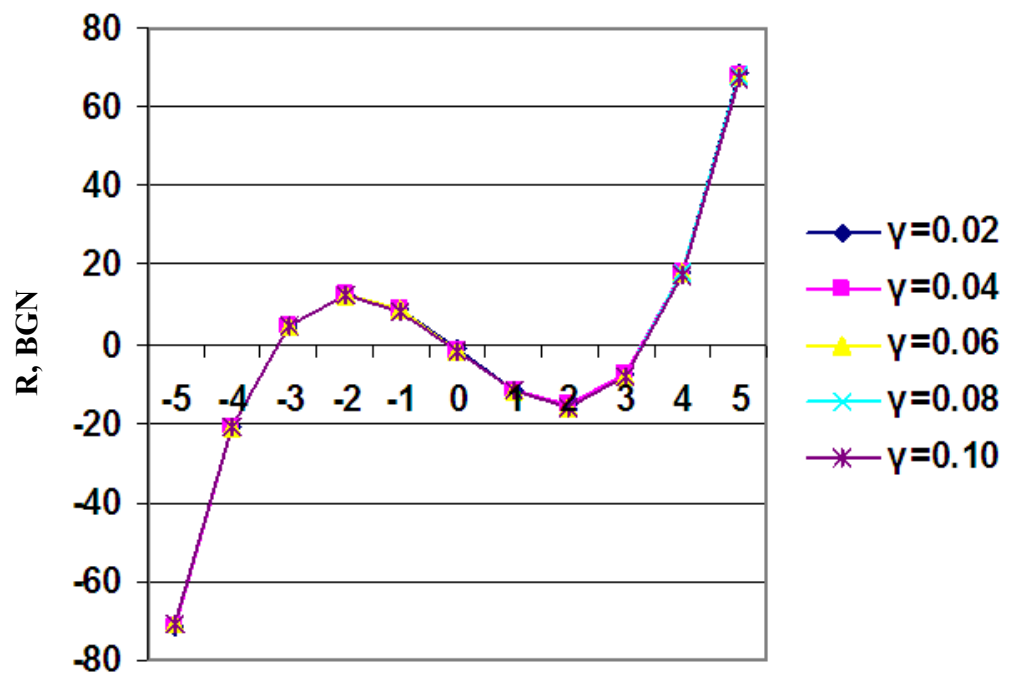

$\mathbf{N}$, number

Fig. 5 The deliveries number $(n)$ fluctuation during the period depending on the coefficient fluctuation $(\gamma)$ by the stock function $\left(Q_{m}\right)$.

\section{Main Conclusions}

A mathematical model for optimization of the necessary SP number for maintenance of the machine workload at an irregular consumption has been elaborated.

The influence of the main parameters of the model over the fluctuation of the SP deliveries' volume number has been studied.

\section{Literature}

[1] Vibe, V., and Sushenko, B. 1981. "On the Method of Rationing Consumption of Tractor Spare Parts." Trudy ChSHI 166: 89-93. (in Russian)

[2] Dimitrov, B. 1984. Scientific Inventory Management-Sofia. (in Bulgarian)

[3] Konstantin, G., Aleksandrov, K., et al. 2002. Survey of Operations-Sofia. (in Bulgarian)

[4] Spiridonov, G., and Tasev, G. 1986. Justification of the Parameters of the System for Distribution of Spare Parts 
between the Units of ROSB. Rousse, Report on the subject No. 8530/1 (not published). (in Bulgarian)

[5] Spyridonov, G., and Tasev, G. 1981. Some Theoretical and Applied Aspects of the Repair and Maintenance of Agricultural Machinery. Rousse. (in Bulgarian)
[6] Hadley, G. 1994. "A comparison of Order Quantities Computed Using the Average Annual Cost and the Discounted Cost." Management Science 10 (3): 472-6.

[7] Harano, H. 1997. "Optimal order quantity NORMS." Operation Research 2 (2): 39-42. 